Supplementary Material for

\title{
Microwave-Assisted Preparation of Hierarchical N and O Co-Doped Corn Cob Derived Activated Carbon for High-Performance Supercapacitor
}

Fangping Wang ${ }^{\mathrm{a}, \mathrm{b}}$, Fenghua Zheng ${ }^{\mathrm{a}}$, Juantao Jiang*a ${ }^{*}$, Yahao Li ${ }^{\mathrm{c}}$, Yingtao Luo ${ }^{\mathrm{b}}$, Kaibin Chen ${ }^{\mathrm{b}}$, Juan $\mathrm{Du}^{\mathrm{b}}$, Youguo Huang ${ }^{\mathrm{a}}$, Qingyu $\mathrm{Li}^{\mathrm{a}}$, and Hongqiang Wang*a

a Guangxi Key Laboratory of Low Carbon Energy Materials, School of Chemistry and Pharmaceutical Sciences, Guangxi Normal University, Guilin, Guangxi 541004, People’s Republic of China

${ }^{b}$ Zhengzhou Non-ferrous Metal Research Institute of Aluminum Corporation of China, Limited, Zhengzhou, Henan 450041, People's Republic of China

${ }^{c}$ State Key Laboratory of Silicon Materials, Key Laboratory of Advanced Materials and Applications for Batteries of Zhejiang Province, Department of Materials Science and Engineering, Zhejiang University, Hangzhou, Zhejiang 310027, People’s Republic of China 

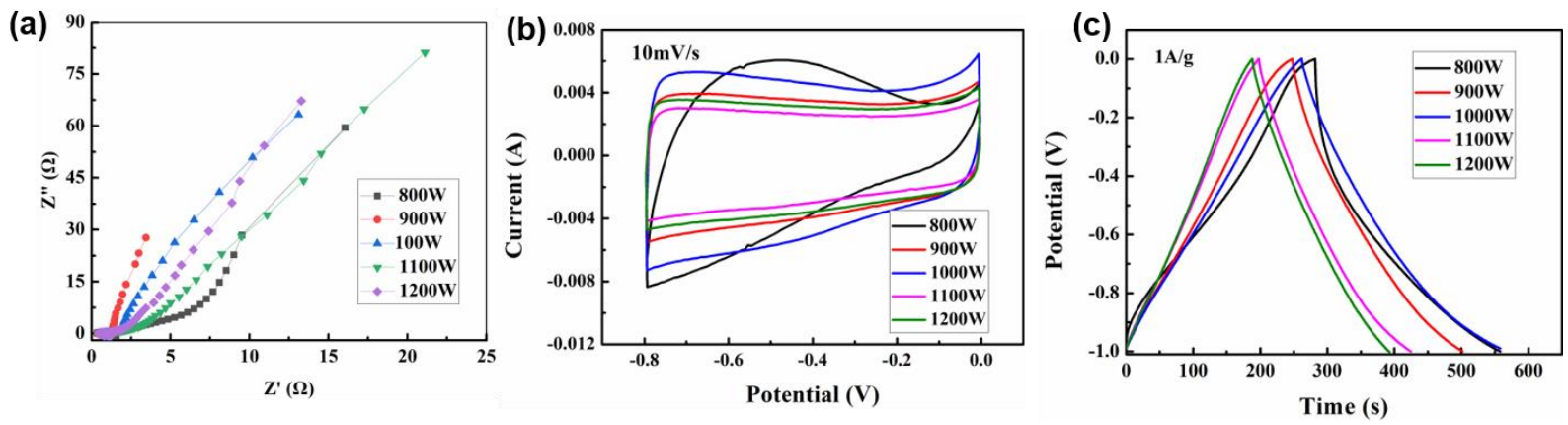

Figure S1. Electrochemical tests of MWAC prepared under different MW powers (Activation time $=30 \mathrm{~min}$, alkali-carbon ratio $=2: 1$ ) in a three-electrode: (a) Nyquist plots; (b) CV curves at scan rates of $10 \mathrm{mV} / \mathrm{s}$ and (c) GCD curves at current densities of $1 \mathrm{~A} / \mathrm{g}$.

Table S1. Specific capacity of MWAC prepared under different MW powers at different current densities (unit: F/g)

\begin{tabular}{cccccc}
\hline & $0.5 \mathrm{~A} / \mathrm{g}$ & $1.0 \mathrm{~A} / \mathrm{g}$ & $2.0 \mathrm{~A} / \mathrm{g}$ & $5.0 \mathrm{~A} / \mathrm{g}$ & $10 \mathrm{~A} / \mathrm{g}$ \\
\hline $800 \mathrm{~W}$ & 354 & 275 & 218 & 155 & 81 \\
$900 \mathrm{~W}$ & - & 253 & 224 & 200 & 192 \\
$1000 \mathrm{~W}$ & 495 & 304 & 258 & 231 & 213 \\
$1100 \mathrm{~W}$ & - & 226 & 203 & 182 & 170 \\
$1200 \mathrm{~W}$ & - & 207 & 188 & 175 & 161 \\
\hline
\end{tabular}



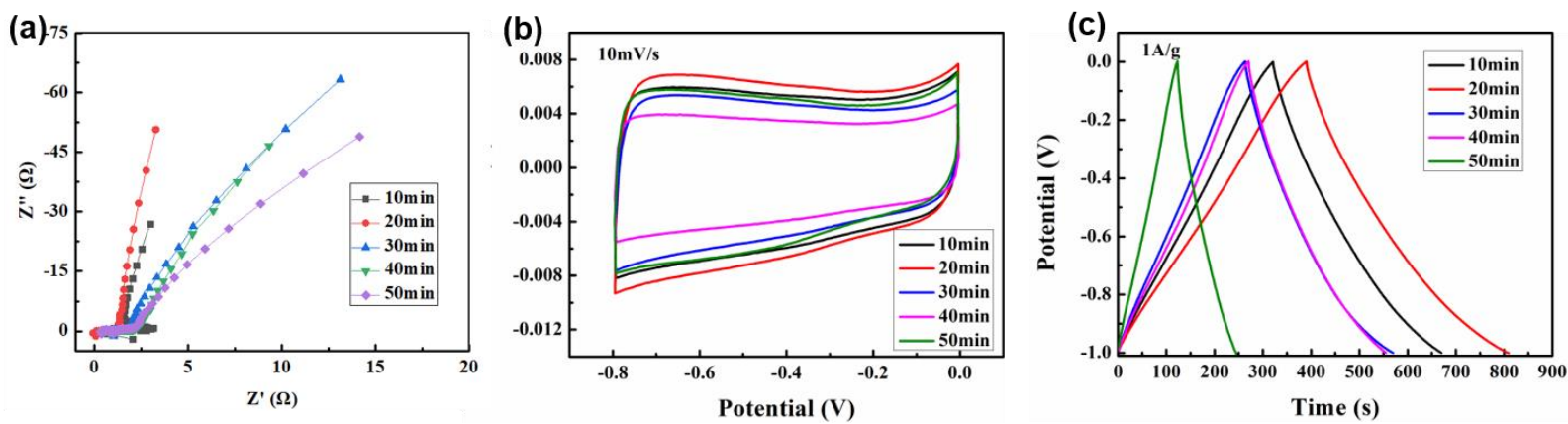

Figure S2. Electrochemical tests of MWAC prepared under different activation times (activation power $=1000 \mathrm{~W}$, alkali-carbon ratio $=2: 1$ ) in a three-electrode: (a) Nyquist plots; (b) CV curves at scan rates of $10 \mathrm{mV} / \mathrm{s}$ and (c) GCD curves at current densities of $1 \mathrm{~A} / \mathrm{g}$.

Table S2. Specific capacity of MWAC prepared under different activation times at different current densities (unit: F/g)

\begin{tabular}{cccccc}
\hline & $0.5 \mathrm{~A} / \mathrm{g}$ & $1.0 \mathrm{~A} / \mathrm{g}$ & $2.0 \mathrm{~A} / \mathrm{g}$ & $5.0 \mathrm{~A} / \mathrm{g}$ & $10 \mathrm{~A} / \mathrm{g}$ \\
\hline $10 \mathrm{~min}$ & 474 & 346 & 308 & 275 & 260 \\
$20 \mathrm{~min}$ & 560 & 414 & 366 & 330 & 300 \\
$30 \mathrm{~min}$ & 495 & 304 & 258 & 231 & 213 \\
$40 \mathrm{~min}$ & 373 & 284 & 252 & 225 & 210 \\
$50 \mathrm{~min}$ & 357 & 273 & 240 & 215 & 200 \\
\hline
\end{tabular}



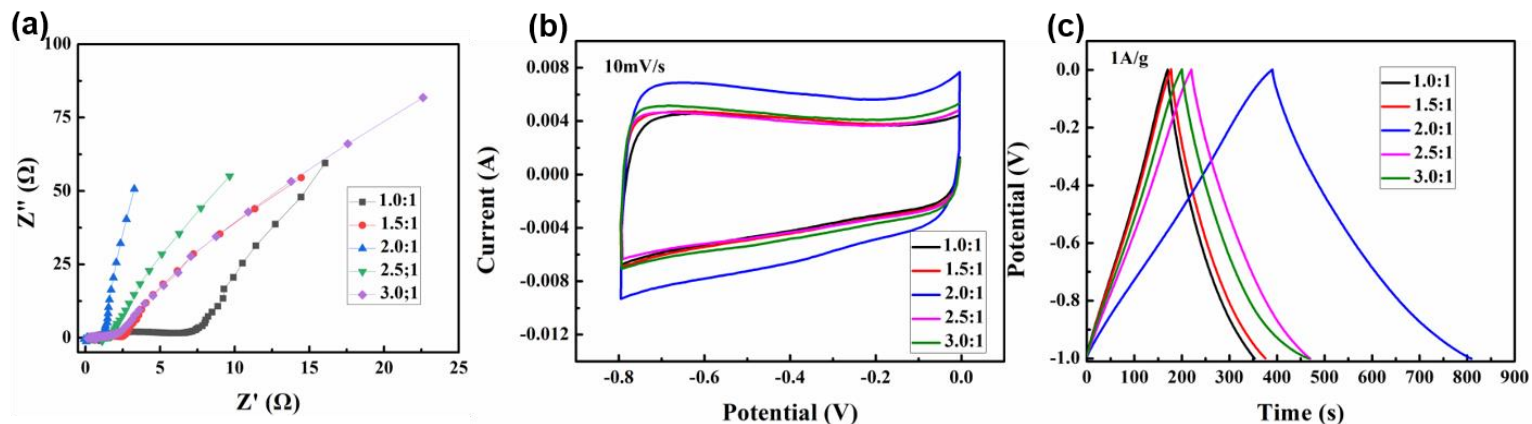

Figure S3. Electrochemical tests of MWAC prepared under different alkali-carbon ratios $($ activation power $=1000 \mathrm{~W}$, activation time $=20 \mathrm{~min}$ ) in a three-electrode: $(\mathrm{a})$ Nyquist plots; (b) CV curves at scan rates of $10 \mathrm{mV} / \mathrm{s}$ and (c) GCD curves at current densities of $1 \mathrm{~A} / \mathrm{g}$.

Table S3. Specific capacity of MWAC prepared under different alkali-carbon ratios at different current densities (unit: F/g)

\begin{tabular}{cccccc}
\hline & $0.5 \mathrm{~A} / \mathrm{g}$ & $1.0 \mathrm{~A} / \mathrm{g}$ & $2.0 \mathrm{~A} / \mathrm{g}$ & $5.0 \mathrm{~A} / \mathrm{g}$ & $10 \mathrm{~A} / \mathrm{g}$ \\
\hline $1.0: 1$ & - & 224 & 183 & 162 & 141 \\
$1.5: 1$ & - & 271 & 198 & 174 & 157 \\
$2.0: 1$ & 560 & 414 & 366 & 330 & 300 \\
$2.5: 1$ & 403 & 336 & 247 & 220 & 195 \\
$3.0: 1$ & - & 292 & 224 & 205 & 181 \\
\hline
\end{tabular}

The systematic condition optimization experiments for the preparation of activated carbon by MW heating method including the microwave power $(800,900,1000,1100$, 
$1200 \mathrm{~W})$, activation time $(10,20,30,40,50 \mathrm{~min})$, and mass ratio of $\mathrm{C}: \mathrm{KOH}(1: 1,1.5: 1$, $2: 1,2.5: 1,3: 1)$ were carried out, and the corresponding results of electrochemical tests are shown in Figure S1, Figure S2, and Figure S3 along with Table S1, Table S2, and Table S3, respectively. These results show that when the MW power $=1000 \mathrm{~W}$, activation time $=20 \mathrm{~min}$, and mass ratio of alkali to carbon $=2: 1$, the capacitor performances of MWAC are best. 